DOI: $10.19195 / 0137-1169.38 .1$

\author{
LESŁAW CIRKO
}

ORCID: 0000-0003-1203-4382

Uniwersytet Wrocławski, Polen

\title{
Emotionen in akademischen Gutachten
}

\section{Einleitung}

\subsection{Ziel und Gegenstand der Untersuchung}

Gegenstand meiner Überlegungen sind Emotionen in Gutachten von Doktorarbeiten und Habilitationsschriften. Das Ziel der Untersuchung besteht darin, Typen von Emotionsäußerungen in einem umfangreichen Korpus quantitativ zu bestimmen und sie danach qualitativ zu charakterisieren.

Akademische Gutachten sind schriftliche Dokumente, die im Redaktionsprozess mehrmals gelesen, stilistisch gefeilt und gattungskonform abgeglichen werden. Man könnte meinen, dass akademische Gutachten angesichts ihrer Spezifik und Zielsetzung emotionsfrei geschrieben werden. Vom Gutachter erwartet man eine professionelle Betrachtung des zu bewertenden Textes. Dazu gehört Sachlichkeit und Unvoreingenommenheit seiner inhaltlich ausgewogenen, stilistisch neutralen Urteile. Das ist die Erwartungshaltung, welche wohl in jeder akademischen Gemeinschaft als selbstverständlich akzeptiert wird.

Am Rande einer großangelegten Korpusanalyse ${ }^{1}$ von akademischen Gutachten (s. in 1.4.) hat sich überraschend herausgestellt, dass ein Teil von fachmännischen Bewertungen affektiv gefärbte Formulierungen enthält. Grund genug, um das Phänomen zu untersuchen.

\footnotetext{
${ }^{1}$ Diese wird im Rahmen des Projekts „INTERDISKURS. Interkulturelle Diskursforschung in akademischen Texten. Vergleichende Studien zur Textorganisation, zu den Formulierungsroutinen und deren Erwerbsphasen in deutschen und polnischen studentischen Arbeiten" durchgeführt. Auf der deutschen Seite wird das Projekt durch die Deutsch-Polnische Wissenschaftsstiftung (Projekt 2014 - 19) und auf der auf der polnischen Seite durch NCN (Nationales Zentrum für Wissenschaft) (Nummer der Entscheidung DEC-2013/08/M/HS2/00044) gefördert. Die Suche nach emotional gefärbten Passagen ist „Nebeneffekt“ der primären Recherche, deren Ziel die Materialsammlung für die Monografie über Standards in philologischen Abhandlungen (Cirko 2020, in Druck) ist.
} 


\subsection{Defizite in der bisherigen Forschung}

Anders als die Textsorte wissenschaftliche Rezension ist akademisches Gutachten (gemeint ist hier ein Dokument im akademischen Promotionsverfahren) bis jetzt kaum untersucht worden. ${ }^{2}$ Das ihm übergeordnete Bewerten ist ein linguistisch relativ gut erforschtes Gebiet, und viele seiner Erkenntnisse können auf das akademische Gutachten übertragen werden. ${ }^{3}$ Die erwähnte Textsorte bleibt aber als autonomes Untersuchungsobjekt nach wie vor ein Brachland in der germanistischen und der polonistischen Forschung. Die Ursachen dafür mögen auf der deutschen Seite darin liegen, dass die zu untersuchenden Texte beschränkt zugänglich sind. ${ }^{4}$ In der Polonistik dagegen hat man offensichtlich bisher kein Interesse an dieser Textsorte gefunden, anders kann man wohl das Ausbleiben der Untersuchungen auf diesem Gebiet nicht erklären.

Emotionen gehören in erster Linie in die Forschungsdomäne der Psychologie. Dort besteht trotz langer und intensiver Auseinandersetzungen nach wie vor kein Konsens in vielen elementaren Fragen. Selbst die Frage, wie viele Basisemotionen es überhaupt gibt, versinkt im Chaos der konträren Argumentationen. Die Autoren schwanken in ihren Angaben zwischen sechs (Ekman 2004) und sechzehn ${ }^{5}$ Emotionen (Plutchik 1980). Darunter gehören zu den meistgenannten: Angst, Scham, Ekel, Freude, Interesse, Traurigkeit, Überraschung, Erwartung und Zorn. Darüber hinaus werden zuweilen auch genannt Glück, Groll, Hass, Liebe, Lust, Not, Panik, Abneigung, Vertrauen, gelegentlich auch andere. Von vielen Forschern werden Emotionen und Gefühle als gleichbedeutend, von anderen

2 Zur Rezension siehe u.a. Jakubeit (1979), Sękowska (2013), Stegert (1997), Zhong (1995), Żmigrodzki (2000). Die Problematik akademischer Gutachten wird (unter einem sehr spezifischen Blickwinkel) bei Cirko (2016) angeschnitten.

${ }^{3}$ Zu dieser Problematik vgl. u. a. Błachut (2012, 2014a), Sandig (1979), Zhong (1995), Zillich (1982).

${ }^{4}$ Von elf Gesuchen an die Dekane philologischer Fakultäten in Deutschland, den Projektmitgliedern (s. oben, Anm. 1) in archivierte Gutachten für Forschungszwecke einen Einblick zu gewähren, blieben sechs unbeantwortet. In fünf weiteren ablehnenden Antworten wurde auf die Möglichkeit verwiesen, Dokumente dieser Art zu sichten, während sie im Dekanat vorschriftsmäßig zur Einsicht vor der Verteidigung/dem Habilitationskolloquium ausgelegt werden, ohne sie - obwohl anonymisiert - für Projektzwecke kopieren zu dürfen. Ähnliches hat der Autor in drei persönlichen Gesprächen erfahren. An dieser Stelle geht mein Dank an alle deutschen Kollegen, die mir ihre elektronischen Gutachtenversionen (insgesamt 27 Exemplare) großzügig zur Verfügung gestellt haben. Die erhaltenen Dokumente bilden kein ausreichendes Gegengewicht für fast 1000 polnische Gutachten, die nun mein Untersuchungskorpus bilden. Die polnischen Gutachten in den gerade laufenden Promotionsverfahren sind nach dem Transparenzprinzip im Netz frei zugänglich. Ein beträchtlicher Teil etwas älterer Gutachten, etwa 2/3, wurde mir durch den Dekan der Philologischen Fakultät in Breslau unbürokratisch zugänglich gemacht, wofür ich ihm an dieser Stelle danke. Die ursprüngliche Absicht, diese Untersuchung kontrastiv anzulegen, hat sich als unrealisierbar erwiesen.

5 Acht Basisemotionen, acht abgeleitete Emotionen. Siehe dazu Plutchiks „Emotionsrad” in Plutchik (1980). 
dagegen als streng zu unterscheidende Erscheinungen betrachtet. ${ }^{6}$ Der zuletzt genannten Option wird gefolgt. ${ }^{7}$

Unzureichend geklärt bleibt auch die Frage, inwiefern Emotionen überhaupt sprachlich ausgedrückt werden können. Es gibt zwar mehrere Publikationen zu dieser Problematik (u.a. Błachut 2012, 2014b, Data 2000, Fiehler 1990, Fries 2000, ein wertvoller Überblick bei Puzynina 2000, Schwarz-Friesel ${ }^{2} 2013$ ), aber die Bedenken bleiben nach wie vor bestehen. Wo ist der Knackpunkt? Emotionen sind primär psychosomatische Erscheinungen: Eine starke innere Regung, die manchmal durch einen einzigen äußeren, oft momentan wirkenden Faktor ausgelöst wird, zeigt sich zunächst einmal im somatischen Bereich (Blutdruckschwankungen, Adrenalinausschüttung, heftiges Erröten oder Verblassen, zitternde Hände, Schwindeln, Schwitzen etc.). Gesichtsausdruck und Körperhaltung sind wie Bildschirme, auf die die Emotionen projiziert werden. ${ }^{8}$ Emotionswallungen spürt man selbst körperlich oder erkennt sie an sichtbaren Körperreaktionen anderer Menschen. Erst dann kann es zu einer (in keinem Fall obligatorischen) sprachlichen Reaktion kommen. Sprache als Mittel zum Emotionsausdruck ist sekundär. ${ }^{9}$ Sekundär, dennoch nach wie vor interessant.

Es sei an dieser Stelle vor möglichen Missverständnissen und Verwechslungen gewarnt: Das Sprechen über Emotionen darf nicht mit emotional gefärbter sprachlicher Ausdrucksweise verwechselt werden, diese wiederum nicht mit Bewertungen, in denen Emotionen mitschwingen, die aber selbst emotional neutrale Ausdrücke sind. Das erste ist - mnemotechnisch - z.B. mit einem beliebten literarischen oder Gesprächstopos zu assoziieren (verzichtet man darauf, ist die ganze populäre Frauenliteratur obsolet, vom Alltagsklatsch ganz zu schweigen!).

6 „Many people use the words 'emotions' and 'feelings' interchangeably, to mean the same thing. However, I suggest that you think of emotions and feelings as distinct, but highly related things - two sides of the same coin. One side of the coin is an emotion: a physical response to change that is almost hard-wired and universal. The other side of the coin is your feeling: mental associations and other reactions to an emotion that are personal, acquired through experience." Vgl. https://emotionaldetective. typepad.com/emotional-detective/2012/04/emotions-vs-feelings.html. Zugriff am 14.5.2019.

7 Zur Begründung vgl. Damasio (1994). Mein Dank geht auch an Frau Dr. Carolyn McNamara-Grant von Ohio Center for Behavioral Psychology, die mir in vielen Laienfragen freundlich in einem geduldigen Gespräch zur Klarheit verholfen hat.

8 „Because emotions are physical, they can be measured objectively by blood flow, brain activity, facial expressions and body stance. Because feelings are mental, they cannot be measured precisely. Emotions are more predictable and easily understood than feelings, which are often idiosyncratic and confusing. Feelings reflect your personal associations to emotions [...]." Vgl. https://emotionaldetective.typepad.com/emotional-detective/2012/04/emotions-vs-feelings.html. Zugriff am 14.5.2019. ${ }^{9}$ Eine andere Perspektive nimmt Fiehler (1990:2-3) ein, indem er von einem methodischen Prinzip ausgeht, ,alle Phänomene so weit wie möglich aus einer interaktionsorientierten und sozialbezogenen Perspektive zu beschreiben und zu erklären und erst dann, wenn diese Erklärungsmöglichkeiten versagen, andere Konzepte - z.B. biologisch-physiologischer Art - in Betracht zu ziehen." In der vorliegenden Arbeit wird anders vorgegangen: Es wird von der zentralen Erscheinung ausgegangen, dann wird ihr Umfeld untersucht. 
Das zweite ist ein affektiver, zuweilen sogar zum Teil unkontrollierter sprachlicher Reflex dessen, was sich tatsächlich in der Psyche des Sprechenden/(seltener) des Schreibenden abspielt. Das dritte gehört zum stilistisch ausgeglichenen Sprachgebrauch ohne emotionellen Hintergrund. Dies sei auf einigen Beispielen aufgezeigt.

1. Zur ersten Gruppe gehören Formulierungen wie Sein Gesicht war plötzlich rot angelaufen, und er ballte seine Fäuste in Wut. Der überwiegende Teil der Fachliteratur, in der das Verhältnis von Sprache und Emotion abgehandelt wird, fokussiert sich gerade auf das Erzählen über Emotionen. ${ }^{10}$ Epische Einsprengsel dieser Art kommen in wissenschaftlichen Bewertungen, die doch keinen narrativen Charakter haben (dürfen), nicht vor.

2. Die zweite Gruppe enthält eine breite Palette von Formulierungsmustern, in denen einerseits Emotionen/Gefühle als begleitende Erscheinungen beim Sprechen/Schreiben namentlich vom Textproduzenten genannt werden, etwa Mit Freude und Belustigung sah ich spielenden Kindern zu. Andererseits die mehr oder weniger spontane sprachliche Manifestationen von Emotionen wie z.B. Igitt, das ist vielleicht eklig!, Aber wieso denn das?!, Du Lump, verdammter!, Du meine Güte!, Ich bringe dich gleich um, wenn du nicht aufhörst.

3. Zur letzten Gruppe werden Formulierungen vom Typ Er hat einen interessanten Essay mit erschreckend vielen, einen Fachmann geradezu beschämenden Fehlern geschrieben. gezählt.

Bei der Eingrenzung des Themas wird auf die Erörterung der Fälle (1) und (3) verzichtet, weil sie kaum etwas mit Emotionen im Sprachgebrauch zu tun haben: (1) benennt physiologisch-psychische Reaktionen eines Menschen, nicht aber seinen Sprachgebrauch, (3) - bewertet, indem er einfach die Attitüden des Bewertenden signalisiert. Ob dabei tatsächlich Emotionen entstehen und von welchen Gefühlen sich der Schreibende leiten lässt, ist nicht nachvollziehbar.

\subsection{Fragestellungen}

Es wird folgenden Fragen nachgegangen:

1. Wie viele untersuchte Texte enthalten affektiv gekennzeichnete Passagen?

2. Welche Typen von affektiven Formulierungen lassen sich im Korpus ermitteln?

\footnotetext{
10 Ein Paradebeispiel dafür ist der 14. Band der in Polen besonders hochgeschätzten Reihe „Język a Kultura“ (2000), wo in kognitivistischer Manier über „Gefühle in Sprache und Text“ (Bandtitel) gesprochen wird.
} 
3. Welche Emotionen hinterlassen sprachliche Reflexe in Gutachten?

4. Inwiefern können die ermittelten Typen als bewusst eingesetzte stilistische Mittel angesehen werden?

Was wird im Folgenden nicht untersucht? Die ermittelten Emotionsäußerungen werden nicht nach ihrem ästhetischen Wert beurteilt. Außerhalb der Betrachtung steht daher die Frage, ob die stilistische Entscheidung des Gutachters richtig, halbwegs richtig oder ganz falsch ist. Weil nur geschriebene Texte analysiert werden, bleiben psychosomatische Reaktionen des Gutachters und jegliche Formen der Emotionsbeschreibung unberücksichtigt.

\subsection{Korpus}

Das Korpus besteht aus 979 polnischen Gutachten von philologischen Dissertationen und Habilitationsschriften aus den Jahren 1997-2019, die im Rahmen des bereits erwähnten INTERDISKURS-Projekts (s. S. 1, Anm. 1) für Forschungszwecke gesammelt wurden. Davon sind 628 Texte Dokumente aus dem Archiv des Dekanats der Philologischen Fakultät an der Universität Wrocław, ${ }^{11} 202$ Gutachten wurden in den Jahren 2013-2019 der offiziellen Internetseite der CK entnommen, ${ }^{12}$ und der Rest (149 Gutachten, teils in elektronischer Form, teils als Printversionen) kommt aus den Privatarchiven von befreundeten Germanisten, Anglisten, Romanisten und Polonisten in ganz Polen, die auf die Bitte um Hilfe bei der Aufstellung eines repräsentativen Gutachtenkorpus positiv reagiert und dem Projektteam ihre Texte zur Verfügung gestellt haben.

\subsection{Methodische Vorgehensweise}

Jedes Gutachten wird vor der Analyse anonymisiert: Namen, Hinweise auf das Thema, Daten und Orte, kurz: Alles, was zur direkten oder indirekten Identifizierung der Gutachter und vor allem der Begutachteten dienen könnte, wird zunächst

\footnotetext{
11 Sie wurden mit Genehmigung des Rektors fotokopiert; alle Kopien werden zurzeit im Archiv des Autors aufbewahrt. Im polnischen Hochschulsystem gibt es in der Regel je zwei Gutachten bei einer Doktorpromotion, je drei bei einem Habilitationsverfahren. Der Leser mag mir eine persönliche Note gestatten: Man verspürt einen Nervenkitzel, wenn man nach Jahren seine (in doppelter Hinsicht) eigenen Gutachten wieder liest; diejenigen, die einem in Promotionsverfahren ausgestellt wurden wie auch die, die man selbst anderen (zuweilen auch für jüngere Institutskollegen) geschrieben hat.

12 Mit $C K$ ist Centralna Komisja do Spraw Stopni i Tytułów [Zentralkommission für Akademische Grade und Titel] gemeint, ein wichtiges Organ beim Ministerium für Wissenschaft und Hochschulwesen. Ab Herbst 2019 wird es kraft Gesetzes durch Rada Doskonałości Naukowej [Rat für Wissenschaftliche Exzellenz] ersetzt.
} 
mit höchster Sorgfalt im Text maskiert. ${ }^{13}$ Danach werden die Texte annotiert; eine von vielen projektrelevanten Optionen ist »Persönliche Töne im Gutachten«. Die unter diesem Blickwinkel annotierten Stellen werden noch einmal gesichtet, Relevantes wird von Zufälligem, Offensichtliches von Undeutbarem getrennt. Die revidierte Liste dient als Basis dieser Veröffentlichung.

Es gibt bekanntlich kein operationales Verfahren, das den untersuchten Äußerungen objektiv und genau einen Wert auf der Achse „nicht emotional $\leftrightarrow \quad$ emotional geprägt“ zuweisen kann. Als Kriterium

bleiben daher die Introspektion und der Rückgriff eines kompetenten Sprechers und seines Sprachgefühls. ${ }^{14}$

Die polnischen Beispiele werden für den deutschsprachigen Leser sinngemäß übersetzt. Bei der Bestimmung von Emotionen und Gefühlen sind ausschließlich polnische Beispiele maßgebend, ihre Form und Lexik, Satzgliedstellung, prosodische Begebenheiten und nicht zuletzt ihre breiteren Kontexte in den untersuchten Gutachten. Eine am deutschen Vergleichsmaterial durchgeführte Analyse wäre eine lohnende Aufgabe (vgl. oben, Anm. 4).

\subsection{Terminologische Erklärungen}

Unter Emotion wird im Folgenden ein zeitweiliger Zustand einer inneren Regung verstanden, die sich primär im somatischen Bereich zeigt, im mentalen Bereich Gefühle auslöst ${ }^{15}$ und dazu noch eventuell kommunikative Reflexe hervorrufen kann. „Kommunikativ“ ist dem Begriff „,sprachlich“ übergeordnet. ${ }^{16}$ Nur sprachliche Reflexe von Emotionen werden in diesem Beitrag behandelt. Mit sprachlichen Reflexen werden Passagen in Gutachtentexten verstanden, die jeweils Emotionen und den von ihnen abgeleiteten Gefühlen zugeordnet werden können.

$\mathrm{Zu}$ den in Erwägung zu ziehenden Emotionen gehören Angst, Scham, Ekel, Freude, Interesse, Traurigkeit, Überraschung, Erwartung und Zorn.

\footnotetext{
13 Namen und Titel werden einheitlich mit XY ersetzt, die übrigen sensiblen Daten werden weggelassen, was mit $[\ldots]$ markiert wird.

14 Dies mag manch einen zur Kritik verleiten, die Untersuchung sei (zu) spekulativ. Den Kontrahenten sei zunächst mit einem Zitat aus einem Gutachten geantwortet: „Spekulation ist wie eine leibliche Schwester der Geisteswissenschaften“ (Übersetzung des Autors). Fragt man einen Generativisten, Korpuslinguisten, Konstruktionsgrammatiker, Rahmensemantiker oder Kognitivisten, ob er im letzten Moment seiner Untersuchung an diesem verflixten introspektiven „Stimmt das, was ich geschrieben habe?" einfach so vorbeigehen kann, muss er meistens zugeben, dass er keine Alternative weiß, und zwar unabhängig davon, wie groß sein antiintrospektiver Anlauf zuvor war. 15 „Despite seeming the same, emotions actually proceed feelings.“ Vgl. https://emotionaldetective. typepad.com/emotional-detective/2012/04/emotions-vs-feelings.html. Zugriff am 14.5.2019.

16 Alle intentional verwendeten Zeichen, die als Vermittlung einer Nachricht (Botschaft) gelten, fallen unter Kommunikation. Sprachliche Kommunikation erfolgt nur mit symbolischen zweiklassigen phonetischen Zeichen oder deren Notationsäquivalenten. Detailliert dazu Cirko (2009:37-51).
} 


\section{Ergebnisse}

Aus der Analyse ergibt sich folgendes Bild:

1. Die Zahl der ermittelten affektiven Passagen beläuft sich auf 111. Sie verteilen sich auf insgesamt 64 Gutachten. 64 Gutachten machen 6,65\% des gesamten Korpus aus. Kehrt man die Perspektive um, kommt man zu dem erfreulichen Schluss, dass über 93\% aller Gutachten hinsichtlich der Emotionsbekundung konventionsgetreu geschrieben werden, und zwar sachlich und neutral.

Tabelle 1: Vorkommen affektiver Passagen im Verhältnis zur Zahl der Gutachten, in denen sie auftreten

\begin{tabular}{|c|c|rr|}
\hline Zahl der Gutachten & $\begin{array}{c}\text { Vorkommen affektiver } \\
\text { Passagen }\end{array}$ & Absolut und prozentual \\
\hline 31 & einmalig & 31 & $(28,0 \%)$ \\
\hline 23 & zweimalig & 46 & $(41,4 \%)$ \\
\hline 8 & dreimalig & 24 & $(21,6 \%)$ \\
\hline- & viermalig & 0 & $(0,0 \%)$ \\
\hline 2 & fünfmalig & 10 & $(9,0 \%)$ \\
\hline & Gesamt & 111 & $(100,0 \%)$ \\
\hline
\end{tabular}

2. Bei affektiv gekennzeichneten Passagen dominieren zwei Formen:

a. einmal Phrasen, in denen auf den psychischen Zustand direkt verwiesen wird, vgl. ${ }^{17}$

$Z$ duża ciekawościa czekatam na rozwinięcie tej tezy i z rozczarowaniem stwierdzam, że czekałam na próżno. 'Mit großem Interesse habe ich auf die Entfaltung dieser These gewartet und mit Enttäuschung stelle ich fest, dass das vergeblich war'.

b. zum anderen Phrasen, die als emotionale, ,spontane“ Reaktionen des Schreibenden gelten, vgl.

Jest to kolejny dowód niestaranności Habilitanta. Po co, po co on to robi? 'Das ist der nächste Beweis der Schludrigkeit des Habilitanden. Wozu, wozu macht er das?‘

Auf die Gruppe (a) entfallen 98 Belege (89,3\% des Korpus), 13 Belege (10,7\% des Korpus) gehören zur Gruppe (b).

17 Die orthografischen und sprachlichen Gegebenheiten des Originals werden immer beibehalten. 
3. Von den neun Basisemotionen, die in Betracht gezogen werden (s. oben, Pkt. 1.2.), hinterlassen Erwartung, Freude, Interesse, Überraschung und Zorn mehr oder weniger deutliche sprachliche Reflexe. ${ }^{18}$

\section{Erwartung:}

Kolejnym logicznym krokiem byloby pogrupowanie uzyskanych danych wedtug klas. Oczekiwanie to zostaje zaspokojone dopiero pod koniec rozdziału 4, w mojej opinii nieco za późno. 'Der nächste logische Schritt wäre, die gewonnenen Daten in Klassen einzuteilen. Diese Erwartung wird erst gegen Ende des Kapitels 4 erfüllt, meiner Meinung nach ein wenig zu spät.‘

\section{Freude:}

Należy z radościa odnotować wielki postęp w badaniach XY nad ta rzadka w Polsce dziedzina badań literackich: od pierwszych nieśmiatych i nieporadnych prób opisu tych ezoterycznych zjawisk poprzez coraz dojrzalsze analizy, a na przedtożonej habilitacji kończac. 'Man soll mit Freude einen großen Fortschritt in den Forschungen von XZ zu dieser in Polen seltenen Domäne literarischer Forschungen registrieren: von den ersten schüchternen und unbeholfenen Versuchen, diese esoterischen Erscheinungen zu beschreiben, über immer reifere Analysen bis hin zu der vorgelegten Habilschrift.

\section{Interesse:}

Nie ukrywam, że moje poczatkowo dosyć umiarkowane zainteresowanie badana problematyka [...] rosto z każda przeczytana strona dysertacji, przekraczajac w pewnym momencie z nawiazka moje wszelkie oczekiwania co do jakości wywodu. 'Ich möchte nicht verhehlen, dass mein anfänglich ziemlich gemäßigtes Interesse an der untersuchten Problematik [...] mit jeder Seite dieser Dissertation wuchs und in einem Moment jegliche Erwartungen hinsichtlich der Ausführungsqualität übertraf.‘

\section{Überraschung:}

Zdumienie budzi brak podania kryteriów, wedtug którego [sic!] następowat dobór podręczników [...] 'Es ist überraschend, dass die Angabe von Kriterien fehlt, nach *dem (korr. denen) die Wahl der Handbücher erfolgte.

18 Eine volle Präsentation der Belege geht über den Rahmen dieses Beitrags hinaus. Viele werden dagegen in Cirko (2020, in Druck) präsentiert. 


\section{Zorn:}

$Z$ rosnaca irytacja odnotowuje wszechobecne $w$ calej pracy niechlujstwo terminologiczne, całkowicie dyskredytujace rozprawe jako tekst o aspiracjach naukowych. 'Mit wachsender Irritation stelle ich eine in der ganzen Arbeit omnipräsente terminologische Schludrigkeit fest, welche die Dissertation als Text mit wissenschaftlichen Ansprüchen völlig diskreditiert. ‘

Die selteneren Emotionsäußerungen vom Typ 2b (vgl. oben) sind nicht eindeutig zu interpretieren. Sie können zuweilen mehreren Gefühlen zugeordnet werden oder lassen die Zuordnung offen.

Zwraca uwage konsekwencja, z jaka XY unika w tym rozdziale precyzyjniejszej charakterystyki cytowanych źródet. Tak nie wolno! 'Es fällt die Konsequenz auf, mit der XY in diesem Kapitel eine präzisere Charakteristik der zitierten Quellen vermeidet. So darf man nicht!' - Zorn?

Z cała pewnościq wyliczenie wszystkich istotnych cech [...] zwieńczyloby ten rozdział. Czyż nie byloby to eleganckim rozwiazaniem, godnym naśladownictwa? 'Ganz gewiss würde eine Aufzählung aller wesentlichen Merkmale von [...] das Kapitel krönen. Wäre das nicht eine elegante, nachmachungswürdige Lösung?' Erwartung?

XY idealnie wyważyt proporcje między poszczególnymi częściami swej obszernej pracy. Brawo! 'XY hat die Proportionen zwischen den einzelnen Teilen seiner/ ihrer umfangreichen Arbeit ideal abgewogen. Applaus!' - Freude?

Welche von den sprachlichen Reflexen echt sind, d.h. der wirklichen Emotion entspringen, kann ohne retrospektive Rückfragen an die Schreibenden kaum bestimmt werden. Übrigens: Eine im Nachhinein gestellte Frage würde wahrscheinlich nichts als einen verdutzten Blick des Befragten zur Folge haben.

4. Aus Gründen, die unten in Pkt. 3, Stichpunkt 4, dargelegt werden, sind die Emotionsäußerungen von Typ (a) und (b), vgl. oben in Pkt. 2, Stichpunkt 2, als bewusst eingesetzte stilistische Mittel in der Textsorte akademisches Gutachten zu betrachten.

\section{Diskussion der Ergebnisse}

Ad 1: Die Zahl der ermittelten affektiven Passagen ist als überraschend hoch zu bewerten. Man kann davon ausgehen, dass die Gutachten mit größter Überlegung 
geschrieben werden und dass ihre Autoren sich der Verantwortung für ihre Qualität bewusst sind, da häufig die akademische Zukunft der Kandidaten davon abhängt. Deshalb entsteht die Vorstellung, dass sich die Autoren davor hüten sollen, emotionale Formulierungen in die Texte einzubauen, es sei denn, sie benutzen sie als bewusst eingesetztes Stilmittel. ${ }^{19}$

Interessant ist die Verteilung der affektiven Passagen. Einmaliges, zweimaliges, ja dreimaliges Vorkommen einer solchen Passage in einem Gutachten ist noch keine verlässliche Grundlage, dies als schreibertypische Eigenart zu deuten. Eine Zäsur bildet die Lücke beim viermaligen Vorkommen, zwei Gutachten mit fünf affektiven Stellen brechen jedoch die Tendenz, die man womöglich zu entdecken glaubt. Hier gibt es wiederum triftige Gründe zur Annahme, dass die Autoren (Rezensenten im Doktorverfahren) ganz absichtlich ihre Texte emotional gestalten. Bei der öffentlichen Dissertationsverteidigung, der in Polen zuweilen mehrere Duzend Zuschauer beiwohnen können, werden Gutachten nämlich vorgelesen. Die Lektüre beider Passagen im breiteren Kontext führt zum Schluss, dass sie einfach affektive Selbstdarstellungsformen sind (mehr dazu in Cirko 2016:154-155).

Ad 2: Eine ungleichmäßige Verteilung der ermittelten Formen $(89,3 \%$ : 10,7\%) zugunsten der namentlichen Bestimmung einer Emotion und der damit verbundenen Gefühle lässt sich durchaus rational begründen. Damit wird eine gewisse Balance zwischen Emotionalität und konventionskonformer Formulierungsweise beibehalten. Etwas trivialisierend lässt sich das so umschreiben: ,ich der Autor/die Autorin bringe meine Gefühle ins Spiel, aber ich habe ständig Kontrolle über sie, indem ich sie direkt benenne und somit nicht (so sehr) gegen das Neutralitätsprinzip im Gutachten verstoße ${ }^{c}$. Die zweite Gruppe von Belegen zeigt dagegen bare Emotionen und Gefühle unvermittelt, d.i. ohne sie zu benennen, wobei ihre Deutung dem Leser überlassen wird. Es entsteht somit eine spezifische Schreiber-Leser-Spannung, die gegen das o.g. Neutralitätsprinzip verstößt.

Ad 3: Vom Autor einer wissenschaftlichen Abhandlung erwartet man, dass er nach all den langen Jahren, in denen er an seinem Opus Magnum gearbeitet hat, ein vollkommenes, komplettes Werk vorlegen wird, das die Fachwelt von Wissen und Können des Autors überzeugt. Aufgrund der Beobachtung, dass zwei im Hinblick auf das Ziel der Gutachten positiv einzuschätzenden Emotionen (Freude, Interesse) zwei eher neutrale (Erwartung, Überraschung) und eine negative, in kritischen Stellungnahmen vorkommende Emotion Zorn gegenüberstehen, ${ }^{20}$ kann vorsichtig die Vermutung aufgestellt werden, dass damit das ganze Begutachtungsspektrum abgedeckt wird.

\footnotetext{
19 Es steht nicht zur Debatte, dass es unzählige Belege für persönliche stilistische Züge im wissenschaftlichen Schrifttum gibt. Sie werden u.a. bei Gajda 1996, Pałucka 2000, Karolczuk 2011 analysiert. Verblüffend ist, dass affektive Formulierungen ausgerechnet in der Textsorte akademisches Gutachten vorkommen, wo emotionale Ausgeglichenheit eine Tugend ist.

${ }^{20}$ Man kann nicht ausschließen, dass eine Untersuchung in einem breiteren Korpus zu einer anderen Verteilung führen würde.
} 
Ad 4: Explizite Bezüge auf die den Gutachter begleitenden Emotionen und Gefühle sowie seltener vorkommende expressive Passagen (vgl. oben in Pkt. 2, Stichpunkt 2b) sind allesamt als bewusst eingesetzte stilistische Mittel einzuschätzen. Stilistische Entscheidungen finden statt, wenn mindestens zwei systemkonforme Ausdrücke um einen Platz im Text konkurrieren. Es liegt dann allein im Ermessen des Textproduzenten, eine der zur Verfügung stehenden Optionen zu wählen. In diesem Moment trifft er eine stilistische Entscheidung (vgl. Vater ${ }^{2}$ 1979:2). Ob sie im jeweiligen Kontext gut oder unangemessen ist, bleibt dahingestellt.

Gutachten entstehen nicht im dynamisch-spontanen Kreationsakt. Ganz im Gegenteil: Die Kommunikationsroutine Beurteilung, zu der sie gehören, setzt mehrere unumgängliche Schritte voraus. Es mag trivial sein: Zunächst muss sich der Gutachter akribisch durch den zu bewertenden Text durcharbeiten (im polnischen Habilitationsverfahren kommen noch viele weitere Unterlagen hinzu!), annotieren, womöglich Exzerpte machen, sein Urteil überdenken etc. etc. Tut er das nicht, ist er kein Profi, kein Experte. ${ }^{21}$ Ein erfahrener Gutachter ist sich auch der Verantwortung dessen bewusst, was er schreibt und wozu: Das von ihm erstellte Dokument kann im Einspruchsverfahren (ein) gegen negative(s) Gutachten vor CK (in Polen immer häufiger auch vor Gericht) als Beweis dienen. Wenn der Gutachter trotzdem zu emotional gefärbten Formulierungen greift, macht er das meist vorsätzlich und ist sich der möglichen Konsequenzen bewusst.

\section{Schlüsse und Ausblick}

In Punkt 3 wurden mehrere Aspekte der affektiven Komponente in der Textsorte Gutachten erwogen. Die Überlegungen legen den Schluss nahe, dass die dort gefundenen Emotionsäußerungen ein seltenes, in der akademischen Alltagsroutine eher unerwartetes, doch vorsichtig und vorsätzlich angewandtes stilistisches Mittel sind. Emotionale Ausdrücke (vgl. oben, 2b) hinterlassen nie den Eindruck, dass sie stilistische Ausrutscher sind (zugegeben eine subjektive Einschätzung).

Emotionsäußerungen vom Typ 2 (vgl. 1.2.), die den Gegenstand der Untersuchung bilden, lassen sich schwer ermitteln: Die angewandte introspektive Methode (vgl. 1.5.) lässt unbestreitbar viele offene Räume für spekulative oder schwer nachprüfbare Schlüsse.

In Gebrauchstexten, der Spezies Gutachten, hier: akademische Gutachten, ist es sehr schwierig, Emotionen zu vermitteln. In der Natur dieser Texte liegt nämlich eine deutliche Dominanz der kommunikativen Funktion über alle anderen. Deshalb wirken sie trocken und sachlich. Die prozentuale Verteilung (ca. 93\% von

21 Dass es bedauerlicherweise in der akademischen Gemeinschaft in Deutschland wie in Polen auch „Zitier-Kartelle“ und „Rezensionstandems“ gibt, weiß man ja seit langem. 
Gutachten im Korpus sind emotional neutral, 7\% weisen Züge der versprachlichten Emotionalität auf; vgl. Pkt. 2, Stichpunkt 1) bestätigt diese Tendenz.

Es entstehen, wie man sieht, Probleme bei der Vermittlung und Ermittlung von Emotionen und Gefühlen in schriftlichen Gutachten. Die gleichen Probleme können in Wirklichkeit auf alle Gebrauchstexte ausgedehnt werden, in denen die kommunikative Funktion über die ästhetische dominiert.

Abhilfe bei der Problemlösung kann vor allem eine saubere Trennung der $\mathrm{Zu}$ ständigkeitsbereiche schaffen: Untersuchungen zum Wesen und zur Manifestation von Emotionen und Gefühlen müssen den Psychologen überlassen werden, die ihr Fachwissen und operationelles Know-how in ihren Laboren besser auszunützen wissen. Die Linguisten müssen dagegen einsehen, dass sie mit ihren philologischen Intuitionen bezüglich der oben erwähnten Erscheinungen allenfalls den in 1.2. unter 1, 2 und 3 umrissenen Bereich untersuchen können, wobei ihre philologisch orientierte Werkstatt bei weitem zu unzureichend ist, um in die Domäne psychologischer Analysen einzudringen.

Was Linguisten hingegen besser als Psychologen können, ist sicherlich ein gewisser „epischer Schwung“ bei der Beschreibung von genuin sprachlichen und sie begleitenden Phänomenen.

Davor müssen die Linguisten aber selbst klären, wo sie die sprachlichen Manifestationen von Emotionen und Gefühlen suchen. Denn Emotionen und Gefühle manifestieren sich generell intersubjektiv in der zwischenmenschlichen Interaktion, oft in der Kommunikation, zuweilen auch in der sprachlichen Kommunikation (=im Sprachgebrauch). In der Sprache dagegen, so wie man diesen Terminus in der Linguistik versteht, gibt es keine Emotionen und Gefühle!

\section{Literatur}

BŁachut Edyta, 2012, Emotionen und Bewertungen sprachlich mitteilen: verbale Ausdrucksmöglichkeiten der emotionalen Einstellung am Beispiel der „taz“-Vuvuzela-Sportkommentare, in: Taborek J./Tworek A./Zieliński L. (Hrsg.), Sprache und Fußball im Blickpunkt linguistischer Forschung, Hamburg, S. 9-24.

BŁachut Edyta, 2014a, Bewerten - Semantische und pragmatische Aspekte einer Sprachhandlung, Hamburg.

BŁACHUt Edyta, 2014b, Einige Bemerkungen zum Verhältnis von Expressivität, Emotionalität, Gefühlswert und Wertungskomponente im aktuellen Sprachgebrauch, in: Łyp-Bielecka A. (Hrsg.), Mehr als Worte. Sprachwissenschaftliche Studien. Professor Dr. habil. Czesława Schatte und Professor Dr. habil. Christoph Schatte gewidmet, Katowice, S. 41-54.

Cirko Lesław, 2009, Akceptacja w komunikowaniu się. Między preskryptywizmem a permisywizmem, Wrocław.

Cirko Lesław, 2016, Między minoderią a ludyzmem: rozważania o kulturze recenzji naukowych, in: Język a Kultura 26, Wrocław, S. 151-161.

Cirko Lesław, (2020, in Druck), Warsztat badacza. Standardy polskiej rozprawy filologicznej na podstawie poradników i recenzji akademickich, Wrocław. 
DAmasio Antonio, 1994, Descartes' Error. Emotion, Reason, and the Human Brain, New York.

DATA Krystyna, 2000, W jaki sposób językoznawcy opisują emocje?, in: Język a Kultura 14, Wrocław, S. 245-252.

Ekman Paul, 2004, Emotions Revealed. Understanding Faces and Feelings, Quezon City.

FIEHLER Reinhard, 1990, Kommunikation und Emotion. Theoretische und empirische Untersuchungen zur Rolle von Emotionen in der verbalen Interaktion, Berlin/New York.

FrIEs Norbert, 2000, Sprache und Emotionen: Ausführungen zum besseren Verständnis; Anregungen zum Nachdenken, Bergisch Gladbach.

GaJDa Stanisław, 1996, Styl osobniczy uczonych, in: Gajda S./Balowski M. (Hrsg.), Styl a tekst, Opole, S. 251-261.

JAKUBEIT Werner 1979, Zur Funktion und Gestaltung der Rezension als einer Textart des Erörterns, in: Textliguistik 7, S. 61-83.

KarolCZUK Agnieszka, 2011, Wybrane cechy idiolektu językoznawcy (na przykładzie Zenona Leszczyńskiego, in: Język a Kultura 22, Wrocław, S. 11-21.

Ludwig Klaus-Dieter, 1974, Zu einigen Fragen des Verhältnisses von Sprache, Wertung und Emotion, in: Sprachpflege 23, S. 51-54.

Patucka Iwona, 2000, O bezemocjonalności tekstów naukowych, in: Ostaszewska D. (Hrsg.), Gatunki mowy i ich ewolucja. Bd.1: Mowy piękno wielorakie, Katowice, S. 147-165.

Plutchiк Robert, 1980, Emotion: Theory, research, and experience: Vol. 1. Theories of emotion, New York.

PuZYNina Jadwiga, 2000, Uczucia a postawy we współczesnym języku polskim, in: Język a Kultura 14, Wrocław, S. 9-24.

SANDIG Barbara, 1979, Ausdrucksmöglichkeiten des Bewertens, in: Deutsche Sprache 2/7, S. 137-159.

Schwarz-Friesel Monika, 2013, Sprache und Emotion (2. Aufl.), Tübingen/Basel.

SĘKOWSKa Elżbieta, Z historii stylu naukowego - początki recenzji językoznawczej, in: Poradnik Językowy 7, S. 21-30.

Stegert Gernot, 1997, Die Rezension: Zur Beschreibung einer komplexen Textsorte, in: Beiträge zur Fremdsprachenvermittlung 31, S. 89-110.

VATER Heinz, 1979, Das System der Artikelformen im gegenwärtigen Deutsch (2. verb. Aufl.), Tübingen.

ZhоNG Lianmin, 1995, Bewerten in literarischen Rezensionen. Linguistische Untersuchungen zu Bewertungshandlungen, Buchframe, Bewertungsmaßstäben und bewertenden Textstrukturen, Frankfurt am Main.

ZILLIG Werner, 1982, Bewerten. Die Sprechakttypen der bewertenden Rede, Tübingen.

ŻmigrodzKi Piotr, 2000, Przemiany czy upadek recenzji językoznawczej? Uwagi metalingwistyczne, in: Ostaszewska D. (Hrsg.), Gatunki mowy i ich ewolucja. Bd. 1: Mowy piękno wielorakie, S. $136-146$.

\section{Internetquellen}

https://emotionaldetective.typepad.com/emotional-detective/2012/04/emotions-vs-feelings. html. 


\section{Emotions in academic opinions}

According to the general opinion, the assessment of scientific dissertations should be emotionally neutral and reliable. However, many evaluations contain emotional expressions. The author argues that linguists have no experience and theoretical basis for speaking about emotions. The author defines the scope of available linguistic research on emotions. The emotions found in the dissertations are analyzed quantitatively and qualitatively on the basis of a large body of evaluations of Polish scientific papers. Linguistic means of expressing emotions are rarely used, but they are also correct stylistic means.

Keywords: scientific dissertation, emotional expressions, limited scope of research, emotions in the evaluations, correct stylistic means. 